\title{
Near-threshold charged kaon pair production in two protons collisions
}

\author{
Damian Gil ${ }^{1, \star}$ and Jerzy Smyrski ${ }^{1}$ \\ for the COSY-11 Collaboration \\ ${ }^{1}$ Jagiellonian University, Cracow, Poland
}

\begin{abstract}
The $p p \rightarrow p p K^{+} K^{-}$reaction was measured near threshold at excess energy $Q=4.5 \mathrm{MeV}$ using the COSY-11 detection system installed at the COSY-Juelich accelerator. Preliminary value of upper limit of the total cross section was determined. This limit indicates that an enhancement of the total cross section close to the threshold, caused by the final state interaction, is smaller than suggested by extrapolation of data measured at higher energies.
\end{abstract}

\section{Physics motivation}

The energy dependence of the $p p \rightarrow p p K^{+} K^{-}$total cross section close to threshold is very sensitive to the interactions between particles in the final state, and therefore is well suited for studies of these interactions. There are many interesting topics connected with the interactions of the final state kaons and protons. For example, the $K^{+} K^{-}$interaction is crucial for understanding the structure of the scalar mesons $f_{0}(980)$ and $a_{0}(980)$, which according Ref. [1,2] are $K \bar{K}$ molecules. In turn, the $K^{-} p$ interaction is interesting from the point of view of study the nature of the $\Lambda(1405)$ hyperon, which is considered as a $K^{-} p$ molecular state [3]. There are also some astrophysical aspects of studying kaons production reactions, like description of neutron stars structure [4]. Elementary reactions of kaon pair production in $p p$ collisions are also important for better understanding of the properties of kaons inside dense baryonic matter [5]. The existing data for the kaon pair production in $p p$ collisions were measured for excess energies higher than $Q=6 \mathrm{MeV}$, with exception of one point at $3 \mathrm{MeV}$, for which only upper limit of the total cross section was determined. On the other hand, data very close to threshold (for $Q<6 \mathrm{MeV}$ ) are crucial for distinguishing between various models of the final state interaction (FSI) in the $p p K^{+} K^{-}$system. Therefore, we performed a high luminosity measurement at $Q=4.5 \mathrm{MeV}$.

\section{Experiment}

The measurement was performed with the COSY-11 detection system, described in details in Ref. [6], at the COSY-Juelich accelerator. A principle of the measurement is presented in Fig. 1. Internal proton beam of the COSY was scattered on the COSY-11 $\mathrm{H}_{2}$ cluster target. The beam momentum

^e-mail: damian.gil@uj.edu.pl 


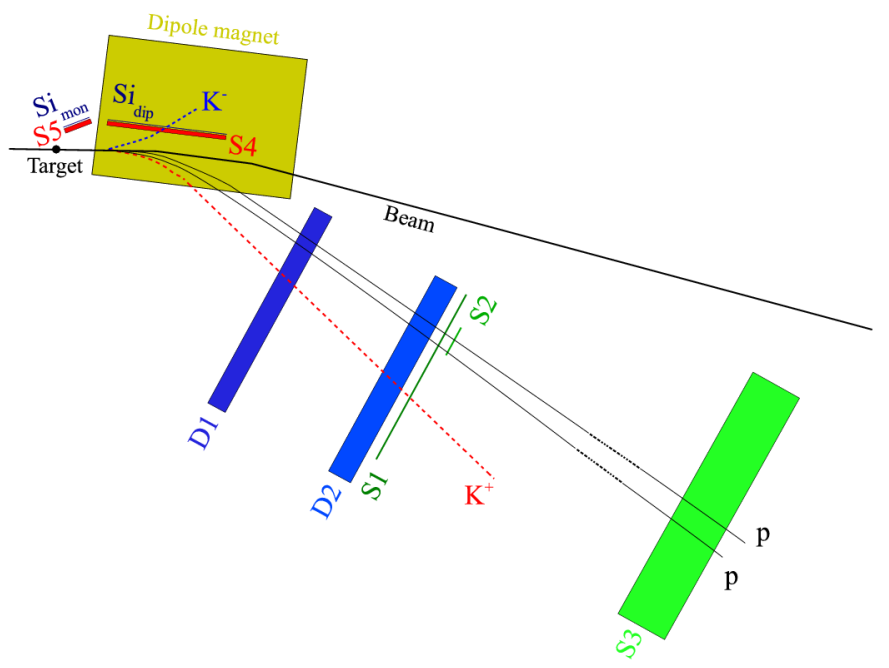

Figure 1. Scheme of the COSY-11 experimental system. Indicated tracks correspond to a simulated $p p K^{+} K^{-}$ event.

was $3.316 \mathrm{GeV} / c$, corresponding to an excess energy in the $p p \rightarrow p p K^{+} K^{-}$reaction of $4.5 \mathrm{MeV}$. With a beam intensity of about $10^{10}$ stored protons and a target density of about $10^{14}$ atoms per $\mathrm{cm}^{2}$, an average luminosity was in the order of $10^{30} \mathrm{~cm}^{-2} \mathrm{~s}^{-1}$. Positively charged reaction products $\left(p p K^{+}\right)$ were deflected outside the COSY ring in one of regular COSY dipole magnets. Their tracks, registered in a set of two drift chambers (D1 and D2 in Fig. 1), were traced back in the magnetic field of the dipole magnet to the target point in order to determine their momentum vectors. Identification of protons was based on the time-of-flight (TOF) measurement on a path of about $9.1 \mathrm{~m}$ between scintillation hodoscopes S1 and S3. For resolving two close proton tracks, a fine granularity hodoscope S2, placed behind the $\mathrm{S} 1$ detector, was also used in the TOF measurements. The positively charged kaons were not registered in S3, and therefore they were identified using TOF on a path between the target and the S1 hodoscope. The luminosity was monitored using a coincidence measurement of the elastically scattered protons. The forward scattered protons were measured in the drift chambers D1 and D2, and the recoil protons were registered in a position sensitive silicon pad detector $\mathrm{Si}_{\text {mon }}$, placed near the target. For identification of the negatively charged kaons, the $p p K^{+}$missing mass was calculated.

\section{Data analysis and results}

Figure 2 presents invariant mass squared of the third positively charged particle registered in the S1 detector, besides the two protons, as a function of the missing mass squared of the $p p K^{+}$system. In the spectrum corresponding to simulations of the $p p \rightarrow p p K^{+} K^{-}$process, a clear signal from the $p p K^{+} K^{-}$events is visible at the invariant mass and the missing mass equal to the $K^{+}$and $K^{-}$mass, respectively. The signal is almost fully confined within $3 \sigma$ limits indicated with red lines in the figure. In the experimental plot, no events are observed within these limits, and therefore only upper limit for the $p p \rightarrow p p K^{+} K^{-}$total cross section can be determined. The background events, visible in the experimental plot, originate mainly from the $p p \rightarrow p K^{+} \Lambda(1405)$ and $p p \rightarrow p K^{+} \Sigma(1385)^{0}$ reactions. The integrated luminosity was determined on the basis of the registered elastic proton-proton counts, normalized to the high precision $p p$ elastic cross section data measured by the EDDA collaboration [7]. In order to validate obtained integrated luminosity, total cross sections for the $p p \rightarrow p p \omega$ and $p p \rightarrow p p \eta^{\prime}$ reactions were determined, based on registered events of the type $p p \rightarrow p p X$. For these events, missing mass spectrum of the $p p$ system contains clear signals corresponding to the $\omega$ 

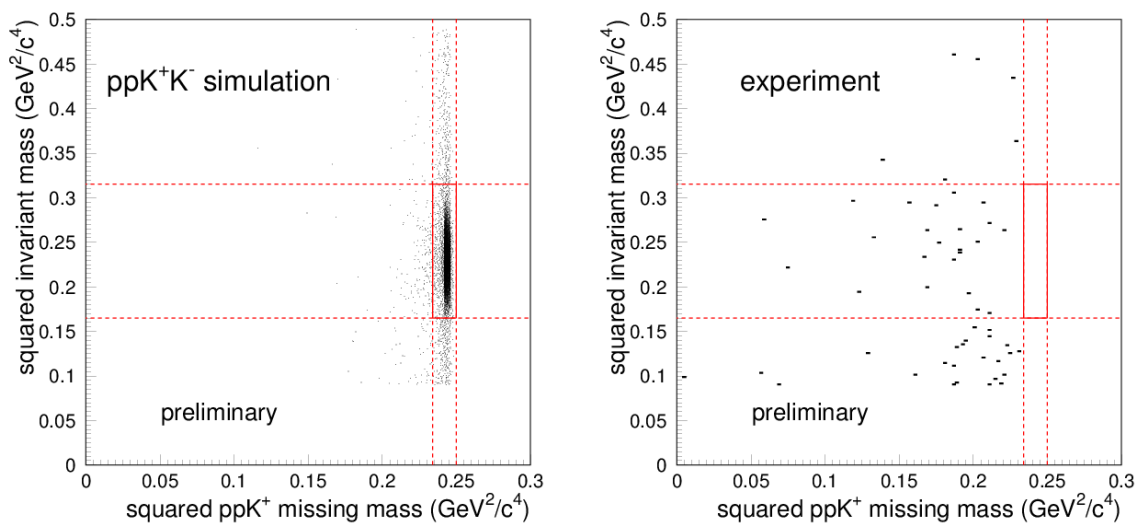

Figure 2. Simulated (left) and measured (right) two-dimensional plots of invariant mass squared of the third (non-proton) particle registered in $\mathrm{S} 1$, versus missing mass squared of the $p p K^{+}$system. Red lines present the $3 \sigma$ limits for distribution of the $p p K^{+} K^{-}$events.

and $\eta^{\prime}$ meson production. Obtained values for the $\omega$ and $\eta^{\prime}$ production cross sections are in a good agreement with results of previous experiments, as shown in Fig. 3. The COSY-11 acceptance for
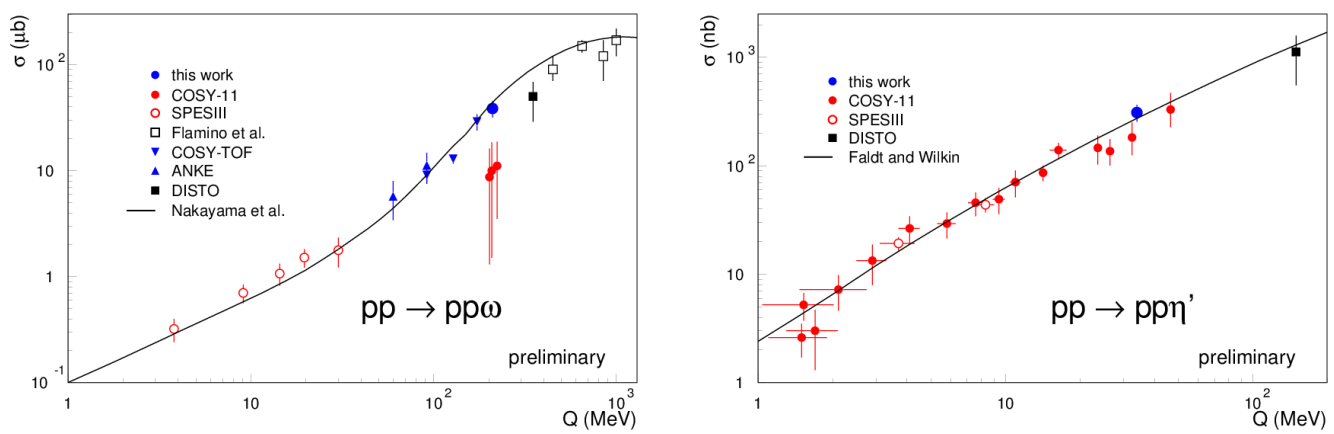

Figure 3. Experimental total cross sections for the $\omega$ production [8-13] and $\eta^{\prime}$ production [11, 14-19], together with the present data points. Solid lines present theoretical predictions for the $\omega$ and $\eta^{\prime}$ production cross sections from Ref. [20] and [21], respectively.

the $p p K^{+} K^{-}$events, determined using Monte Carlo simulations taking into account the $p p$-FSI, is equal to $2.83 \%$. Preliminary upper limit for the $p p \rightarrow p p K^{+} K^{-}$total cross section at $Q=4.5 \mathrm{MeV}$, calculated using the obtained integrated luminosity and the acceptance, is presented in Fig. 4, together with existing experimental data. As shown in the figure, energy dependence of the experimental data points is not reproduced by the four-body phase space integral. Inclusion of the $p p$-FSI still underestimates the experimental values. Also inclusion of the $p K^{-}$-FSI, parameterized by the scattering length approximation with $a_{p K^{-}}=(0+1.5 i) \mathrm{fm}$, according Ref. [22], underestimates the experimental results, especially in the low energy region. Only after including an extra $K^{+} K^{-}$-FSI factor, parameterized 
with the effective range approximation with $a_{K^{+} K^{-}}=(8+0 i) \mathrm{fm}$ and $b_{K^{+} K^{-}}=(-0.1+1.2 i) \mathrm{fm}$ [23], the previous data points are well described, but the present upper limit of the cross section is overestimated.

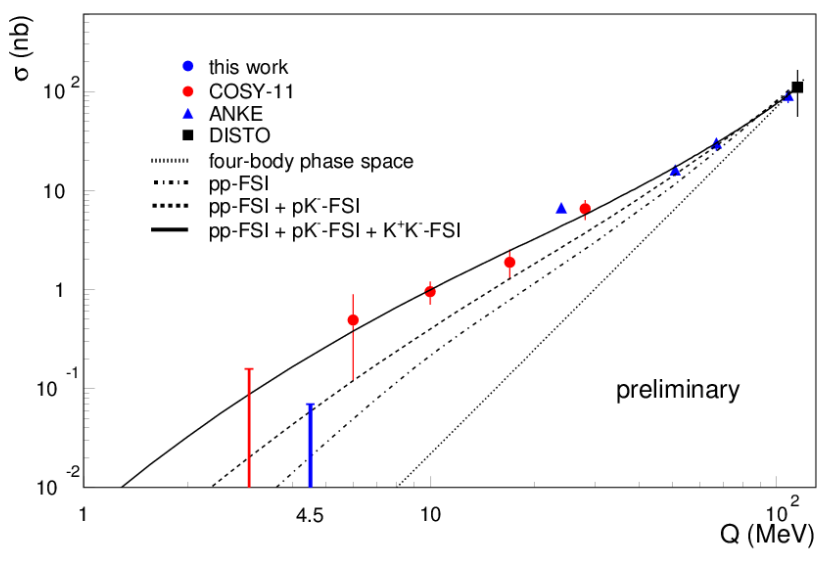

Figure 4. The $p p \rightarrow p p K^{+} K^{-}$ excitation function obtained from previous experiments [11, 24-28], together with the present upper limit of the total cross section. The dotted line presents an energy dependence according the phase space volume, the dotted-dashed line incorporates the $p p$-FSI, the dashed line includes additionally the $p K^{-}$-FSI and solid line is obtained by taking into account also the $K^{+} K^{-}$interaction. All theoretical calculations are normalized to the DISTO point at $Q=114 \mathrm{MeV}$.

\section{Conclusions}

We measured the $p p \rightarrow p p K^{+} K^{-}$reaction at $Q=4.5 \mathrm{MeV}$ using the COSY-11 detection system. With no $p p K^{+} K^{-}$observed events, we determined only upper limit of the total cross section. The present limit lies below theoretical calculations, including FSI factors for $p p, p K^{-}$and $K^{+} K^{-}$systems, reproducing well experimental data measured at higher energies. This deviation can result from the Coulomb interaction, which was not fully included in the theoretical calculations. It can be also caused by the strong $K^{+} K^{-}$absorption due to formation of hypothetical $K \bar{K}$ molecule [29].

\section{References}

[1] M. Bargiotti et al., Eur. Phys. J. C 26, 371 (2003)

[2] N.N. Achasov and G.N. Shestakov, Phys. Rev. D 58, 054011 (1998)

[3] J.M.M. Hall et al., Phys. Rev. Lett. 114, 132002 (2015)

[4] Y. Lim et al., Phys. Rev. C 89, 055804 (2014)

[5] P. Moskal et al., J. Phys. G 28, 1777 (2002)

[6] S. Brauksiepe et al., Nucl. Instr. and Meth. A 376, 397 (1996)

[7] D. Albers et al., Eur. Phys. J. A 22, 125 (2004)

[8] V. Flaminio et al., Compilation of Cross-Sections, CERN-HERA, 84 (1984)

[9] F. Hibou et al., Phys. Rev. Lett. 83, 492 (1999)

[10] F. Balestra et al., Phys. Rev. C 63, 024004 (2001)

[11] C. Quentmeier et al., Phys. Lett. B 515, 276 (2001)

[12] S. Abd El-Samad et al., Phys. Lett. B 522, 16 (2001)

[13] M. Abdel-Bary et al., Phys. Lett. B 647, 351 (2007)

[14] P. Moskal et al., Phys. Rev. Lett. 80, 3202 (1998)

[15] F. Hibou et al., Phys. Lett. B 438, 41 (1998) 
[16] P. Moskal et al., Phys. Lett. B 474, 416 (2000)

[17] F. Balestra et al., Phys. Lett. B 491, 29 (2000)

[18] A. Khoukaz et al., Eur. Phys. J. A 20, 345 (2004)

[19] P. Klaja et al., Phys. Lett. B 684, 11 (2010)

[20] K. Nakayama et al., Phys. Rev. C 60, 055209 (1999)

[21] G. Fäldt and C. Wilkin, Phys. Rev. C 56, 2067 (1997)

[22] Y. Yan, arXiv:0905.4818

[23] M. Silarski et al., Phys. Rev. C 88, 025205 (2013)

[24] M. Wolke, Ph.D. thesis, Rheinische Friedrich-Wilhelms-Universität Bonn (1997)

[25] F. Balestra et al., Phys. Lett. B 468, 7 (1999)

[26] P. Winter et al., Phys. Lett. B 635, 23 (2006)

[27] Y. Maeda et al., Phys. Rev. C 77, 01524 (2008)

[28] Q.J. Ye et al., Phys. Rev. C 87, 065203 (2013)

[29] C. Wilkin, private communication (2016) 